\section{At the end of the rainbow -}

\section{an understanding of nuclear matter}

\author{
W. von Oertzen, Dao Tien Khoa, H.G. Bohlen \\ Hahn-Meitner-Institut Berlin and Fachbereich Physik, FU Berlin
}

$F_{\text {airy tales tell us of the rainbow as the }}$ mystery resides in the wave properties of light in the properties of water (refractive index) and the spherical shape of water droplets. At a deeper level our microscopic knowledge of the properties of water molecules will be needed in order to understand the refraction of light waves.

The accumulation of many light rays into a narrow angular region around the rainbow angle which is at the heart of this phenomenon needs a sophisticated mathematical description. The mathematical challenge has triggered intense intellectual activity over centuries, ever since Descartes $\left({ }^{*} 1694\right)$. The same phenomenon is found in the scattering of particles, which also have wave properties, and the world of quantum mechanics opens up, giving access to the microscopic description of the interaction between complex particles like atomic nuclei.

This short account combines two related topics which have been on the forefront of research in nuclear physics in the last decades. These studies aim, i) at the determination of nucleus-nucleus potentials from scattering data and, ii) the determination of the properties of nuclear matter dictated by the equation of state. To be more specific - the value of the bulk modulus $K$, the incompressibility of cold nuclear matter, which characterises the "stiffness" of matter to higher densities, is determined.

Nuclei are massive composite objects, which are subject to quantum mechanical descriptions for their motion as a whole and of their constituents. The short range properties of nuclear forces, which have been well studied over recent decades, gives rise to the saturation of the binding energy at the so called saturation density $\rho_{0}$. Nuclei are pieces of nuclear matter and can give clues to the equation state of extended nuclear matter through the dependence of the total energy on the nuclear density. We study the elastic channel in nuclear scattering because it is a unique and well defined observable, connected to the ground states of nuclei, well known both

experimentally and theoretically, and information from elastic scattering can be related to the properties of cold nuclear matter $[1,2]$.

The study of nuclear matter, as well as the modelling of the properties of stable nuclei, their radii, their binding energies, level schemes, etc. relies on the knowledge of the nucleon-nucleon-interaction inside the nuclear medium, also referred to as an effective interaction between nucleons. We will see later that the study of refractive nuclear rainbow scattering, where massive composite particles interact and penetrate each other, is a unique source of information on this interaction of nucleons inside nuclear matter.

The scattering of massive particles is described by their wave properties, which are determined by the de Broglie wavelength. This is given by the energy, $E$, and the mass (reduced mass), $\mu$, of the particle as, $\lambda=\hbar /(2 \mu E)^{1 / 2}$. The "asymptotic"kinetic energy is $E_{o}$ at large distances. Another part of the energy is due to the potential $V(r)$ created by the interaction with the medium, $E=\left[E_{o}+V(r)\right]$. Refractive scattering of particles travelling through matter can be described in terms of a refractive index, $n=\lambda_{0} / \lambda$, given by the ratio of the wavelengths, or by

$$
n(r)=\sqrt{\left(E_{o}-V(r)\right) / E_{o}}
$$

The refractive scattering of nuclear particles is mostly related to attractive potentials with $V(r)<0$, where $E>E_{0}$ and hence to a refractive index $n$ larger unity.

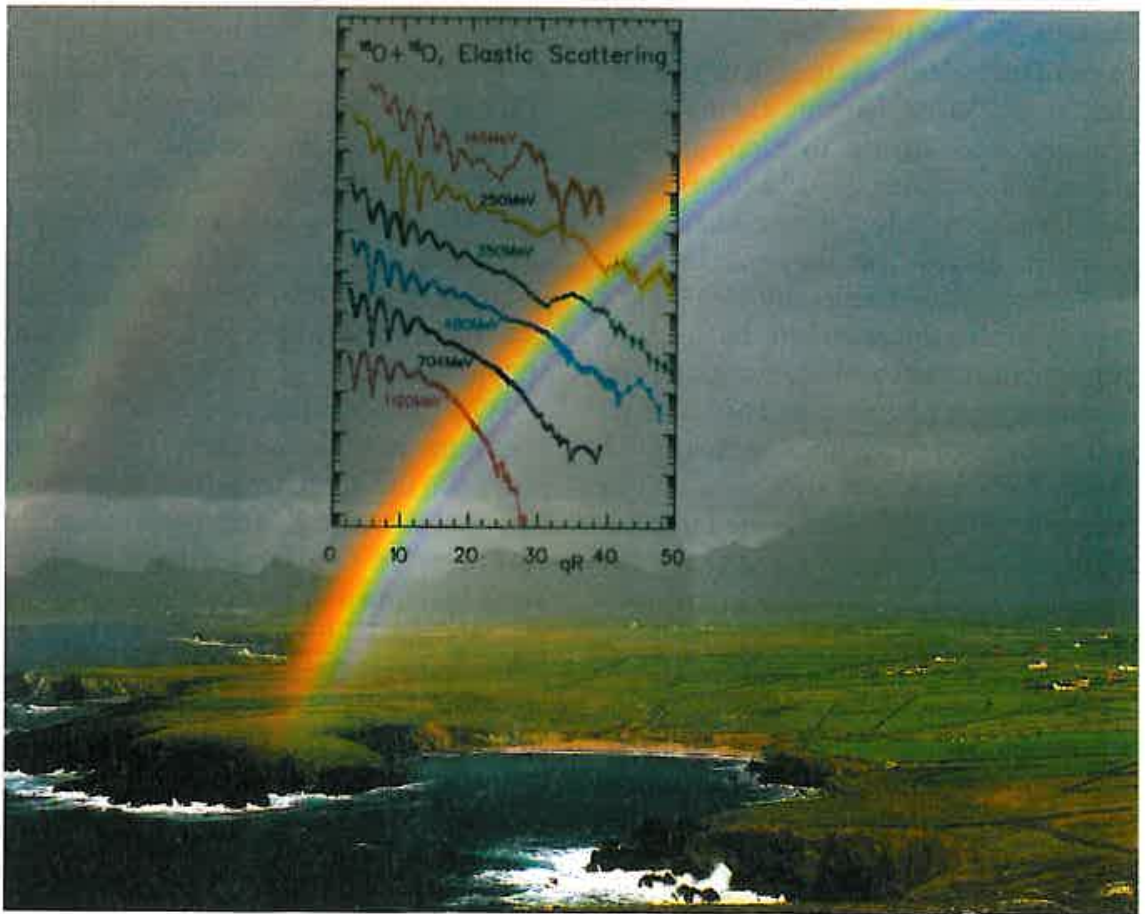

Fig. 1 Example of diffractive and refractive elastic scattering in the ${ }^{16} \mathrm{O}+{ }^{16} \mathrm{O}$ system over a wide range of energies. Note that the ratio of the differential cross section to Mott is plotted as function of the momentum transfer, $q=\left|\vec{k}_{i}-\vec{k}_{f}\right|$, multiplied by the interaction radius $R$. In this case the diffractive pattern appears unchanged over many energies, whereas the refractive pattern (the rainbow) moves as function of energy.

The primary rainbow maximum moves to larger angles with decreasing energy and moves beyond $\theta_{C M}=90^{\circ}$ for $E_{L}<250 \mathrm{MeV}$. There the Mott interference pattern for the identical Bosons, ${ }^{16} \mathrm{O}+{ }^{16} \mathrm{O}$, is responsible for the oscillations around $\theta_{C M}=70^{\circ}-90^{\circ}$. 


\section{Diffractive and Refractive Scattering}

The waves associated with nuclear particles are not only refracted (changing their wavelength and direction) but usually also absorbed, giving rise to diffraction. Diffraction occurs if a geometrical object - a sphere in our case removes flux from the incoming waves. The corresponding intensity pattern as function of scattering angle, observed at far distances is known as Fraunhofer diffractive scattering. The pattern shows intensity maxima which are separated by very sharp minima; the distance in angle between these minima is determined by the radius of the object, $R$ (or the sum of two radii), and the wavelength, $\lambda_{0}$. Examples of this behaviour are seen at small angles in cases shown in figures 1 , and 2.

Nuclear scattering at energies, for which the Coulomb forces are relatively weak, show pronounced Fraunhofer diffractive patterns at small scattering angles, because strong absorption sets in within a range $a \approx 0.6 \mathrm{fm}$, which is small compared to radii of $R \approx 5 \mathrm{fm}$. The unique property of the diffraction process, the existence of very sharp intensity minima, can be seen if

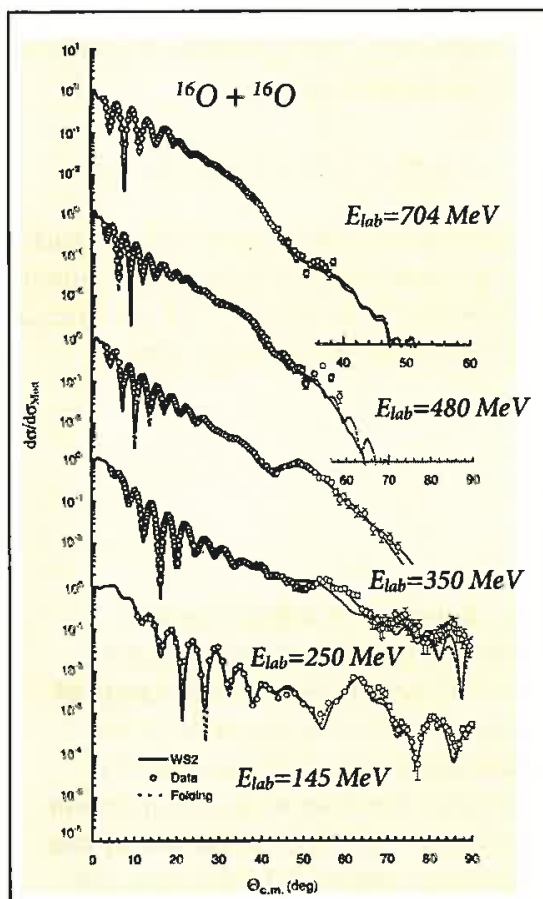

Fig. 2 A choice of differential experimental cross sections for elastic scattering of ${ }^{16} \mathrm{O}+{ }^{16} \mathrm{O}$ (divided by Mott scattering) with curves calculated using the double folding model and density dependent nucleon-nucleon forces. Note the different scales of the angle co-ordinates for different energies. the scattering pattern is plotted as function of the difference $q$ of the wavenumbers

$$
q=\left|\bar{k}_{i}-\bar{k}_{f}\right|=2|k| \sin \frac{\theta}{2}
$$

as done in fig. 1. In this case the minima remain fixed at a certain value of $q R$ independent of incident energy. Actually the radius $R$ of the "black" object is multiplied to obtain the variable $q R$ in fig. 1 .

First observations of refractive and rainbow phenomena in nuclear scattering were made by Goldberg and Smith 25 years ago in elastic scattering of $\alpha$-particles on $\mathrm{Ca}$ targets in the energy range of $20-30 \mathrm{MeV} /$ nucleon. An important aspect of the $\alpha$-nucleus scattering is the reduced absorption of the particles penetrating the target nucleus. This is caused by the large binding energy of nucleons in ${ }^{4} \mathrm{He}$ and the saturation of the nucleon-nucleon forces in cases of projectiles and target nuclei with closed shells. In nucleus-nucleus scattering, ${ }^{16} \mathrm{O}+{ }^{16} \mathrm{O}$ has become the system of preference, because of the tight binding at the closure of the p-shell in ${ }^{16} \mathrm{O}$. The observation of the first Airy-maximum [see Box 1] as a clear rainbow-effect in the ${ }^{16} \mathrm{O}+{ }^{16} \mathrm{O}$ system at an energy of $E_{L}=350 \mathrm{MeV}$ has been established in 1989 at HMI Berlin. The density dependence of the nucleon-nucleon interaction underlying the nucleus-nucleus potential can be determined from such nuclear rainbow scattering, because density overlaps, with twice the saturation density $\left(\rho_{o}\right)$ of nuclear matter, occur in some part of the overlap region [3].

\section{Nucleus-Nucleus potentials}

In refractive elastic scattering of two nuclei, two ${ }^{16} \mathrm{O}$-nuclei for example are brought into strong overlap in their ground states during the collision process. For elastic scattering at intermediate energy we can assume that the densities overlap without disturbance, and we obtain a density profile for small internuclear distances, which reaches twice the saturation density of nuclear matter $\rho_{0}$. This value, $\rho_{0}$, is also observed in the interior part of ${ }^{16} \mathrm{O}$. This feature of density overlap is unique for a quantal system (not related to compression, which would simultaneously heat up the system). Nucleons interact at a higher local density in the overlap region, and the effects of antisymmetrisation will appear in different exchange processes. The double folding model $[2,3]$ for the calculation of the potentials uses the two frozen densities $\rho_{1}(r)$ and $\rho_{2}(r)$, folded with a properly chosen effective nucleonnucleon interaction $V_{\text {eff }}=V_{N N}\left(\left|r_{1}-r_{2}\right|\right)$.
The matrix elements calculated in the double folding model are equivalent to those encountered in Hartree-Fock calculations in the local density approximation, where $V_{\text {eff }}$ has an exchange term. The folding model can be considered to be a semimicroscopic approach, because no free parameters (except for an overall normalisation) enter in the calculation. The necessary information is the following: $i$ ) two densities of ${ }^{16} \mathrm{O}$, taken from electronscattering, they are represented by appropriately chosen harmonic oscillator wave functions, and ii) the nucleon-nucleon interaction, $V_{N N}$, which is derived from a Gmatrix describing nucleon-nucleon scattering phase shifts, and describing well average nuclear properties. iii) Finally a density dependence is introduced for $V_{N N}$, which is chosen to reproduce the well known saturation properties of nuclear matter. We may already conclude that the interaction of nucleons in their density dependence can be probed via the nucleusnucleus potentials if the nuclear rainbow is observed, which scans the nucleus-nucleus interaction at small distances. The density dependence of $V_{N N}(r)$ enters in the determination of the saturation properties of nuclear matter in Hartree-Fock calculations [Box 2], we can thus foresee that the study of refractive nucleus-nucleus potentials will give a unique access to the study of the equation of state of cold nuclear matter.

By the choice of parameters of the effective interaction, "soft" or "stiff" nuclear matter, can be obtained in the calculations.

There appears an exchange term $V_{e x}(r)$ in the nucleon-nucleon interaction, which enters also in the Hartree Fock calculations for nuclear matter; the exchange term is non-local and contains the relative momentum of the nucleons, which in turn depends on the nucleus-nucleus potential created during the overlap. Thus the calculation of this term has been done selfconsistently using appropriate numerical iterations.

With the density dependence of the inmedium nucleon-nucleon interaction chosen in our approach so as to reproduce different values of $K$, we can directly relate "stiffness" in the Hartree-Fock calculations [as shown in Box 2] to the result of the double folding potentials; the potentials are expected to be less deep (i.e. in the region of large density overlap) for a more stiff equation of state, because more repulsion is obtained at higher densities.

The ${ }^{16} \mathrm{O}+{ }^{16} \mathrm{O}$ System

In the first clear observation of a nuclear 


\section{Box 1 Rainbows}

For the understanding of the refractive scattering and the rainbow phenomenon we have to discuss the deflection function, which describes the variation of the deflection angle, $\theta=f(b)$ with the impact parameter $b$ (the distance of the incident particle from the central axis, as illustrated in fig. B1.1). The well known optical rainbow, which occurs after refraction and one reflection of the light beam in the water droplet, has been correctly interpreted by Descartes, who was the first to realise the importance of the caustic, the accumulation of lightrays observed at the maximum deflection angle. The deflection function for light in a waterdroplet is shown in fig. B1.1 together with a case of nuclear scattering, $\left({ }^{16} \mathrm{O}+{ }^{16} \mathrm{O}\right)$, discussed in the main text

The rainbow phenomenon places an increased light intensity at the rainbow angle, $\theta_{R}$, because a finite region of impact parameters contributes to differentially small regions of the deflection angle at $\theta_{R}$. The position of the rainbow angle, $\theta_{R}$, itself depends on the wavelength, $\lambda$, because it is related to refraction, giving rise to a splitting of colours for a white spectrum of incident waves. The maximum deflection angle would define a sharp boundary between light and shadow - in classical optics, the intensity would diverge at this angle as shown in fig. B1.2. The solution of the mathematical problem is difficult and has been solved in the middle of the 20th century [1]. The results of some mathematical approaches are illustrated in fig. B1.2. The correct mathematical description of the intensity variation was first introduced by Airy, defining the Airy function. The first intensity-(Airy) maximum appears slightly shifted just inside the classical illuminated region followed by the first Airy minimum (A1) and by further Airy maxima and minima. We will refer to these as the first, second, third etc. Airy maxima or minima. They are observed in the data on ${ }^{16} \mathrm{O}+{ }^{16} \mathrm{O}$ scattering at the lower energies of $80-150 \mathrm{MeV}$.
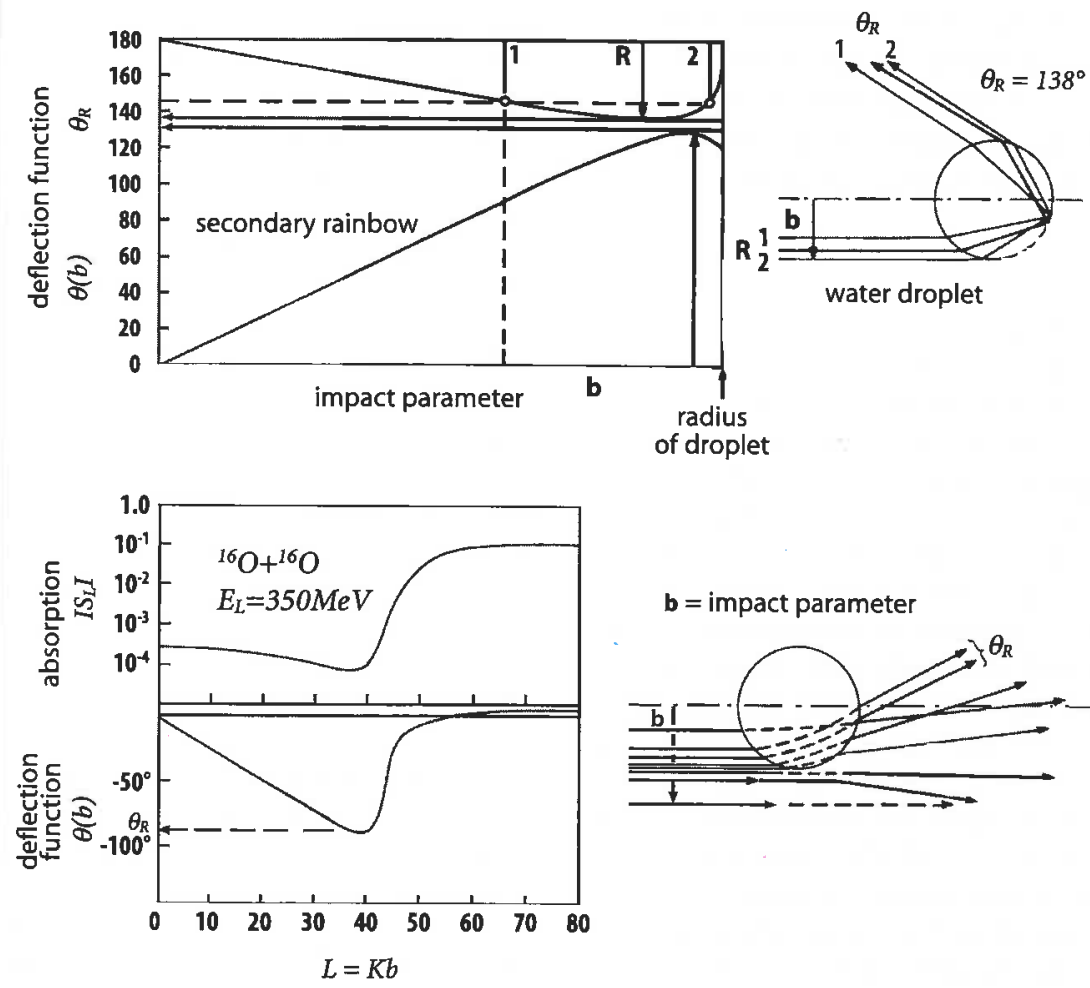

b = impact parameter

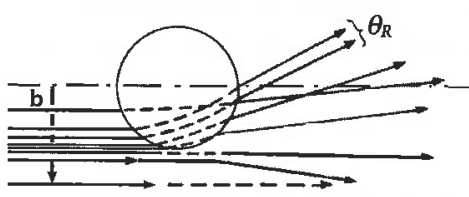

Eig. B1.1 Deflection functions for the case of, a) the light scattering on water droplets. In this case the deflection angle varies from total reflection at $\theta=180^{\circ}$, down to $\theta_{R}=138^{\circ}$, with one reflection inside. A secondary rainbow appears (see fig 1) with a second reflection in the water droplet. In case, b) of ${ }^{16} \mathrm{O}+{ }^{16} \mathrm{O}$ elastic scattering at an energy of $30 \mathrm{MeV} /$ nucleon attractive potentials give rise to negative deflection angles with a maximum at $\theta_{R}$; the rainbow angle $\theta_{R}$

\section{Look for ${ }_{\text {LETIERS }}^{\text {EUROPYSSII }}$ \\ Please look to}

Vol. 49, Num. 5, 1

March 2000 of

EUROPHYSICS

LETTERS to learn

more about the

following:

Quantum halos -

K. Riisager et. al. $\cdot A$

robust perturba-

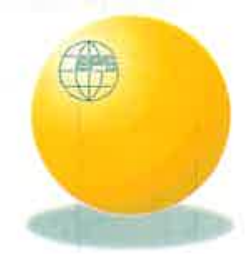

tion theory for degenerate states based on exact constants of the motion - E. Oks \& T. Uzer - Hydrogen-anti-hydrogen elastic scattering using fully quantal method - P.K. Sinha \& A.S. Ghosh - Laser beam self-phase modulation by a film of water-in-oil microemulsion - L.Vicari - The critical temperature for structural recovery of amorphous glassy polymers - A. D. Drozdov - Analysis of compressibility effects on Darrieus-Landau instability of deflagration wave $-\mathrm{L}$. He -Wetting transition of a binary liquid mixture at a solid boundary - A. Plech et. al. - Aging as dynamics in configuration space - W. Kob et. al. - EXAFS and Molecular Dynamics combined study of glass. New insight into site significance in silicate glasses - S. Rossano et. al. - Quasicrystalline gold interface with a hypo-friction property - F. Lançon et. al. - Low-frequency dynamics in molecular incommensurate composite: Specific heat of nonadecane/urea inclusion compound - J. Etrillard et. al. - Theory of the island and capture zone size distributions in thin film growth - P. A. Mulheran \& D. A. Robbie - The surface composition and spin polarization of NiMnSb epitaxial thin films - D. Ristoiu et. al. • Enhanced thermal dissociation of optically excited chains - P.-A. Persson et. al. - "Excitoner": Stimulated amplification and propagation of excitons' beams - A. R. Vasconcellos et. al. Dynamic critical behaviors of three-dimensional $X Y$ models related to superconductors/superfluids - L. M. Jensen et. al. • Nano-patterning of magnetic anisotropy by controlled strain relief - W. Wulfhekel et. al. Gyromagnetic ratio and magnetization in Fe/V superlattices - M. Farle et. al. • Resonant mixing of widely separated intermediate states and charge transfer at the 4d-4f resonance of La compounds - A. Moewes et. al. Liquid-solid transition of Laponite suspensions at very low ionic strength: Long-range electrostatic stabilisation of anisotropic colloids - P. Levitz et. al. - The fluid-fluid interface of a model colloid-polymer mixture - J. M. Brader \& R. Evans - Statistical mechanics of mutual information maximization - R. Urbanczik 
decreases with increasing energy. For ${ }^{16} \mathrm{O}+{ }^{16} \mathrm{O}$ scattering the absorption at small impact parameters, (given as orbital angular momentum $L=K b(K=$ wavenumber)) is shown by the function $\left|S_{L}\right|$ which is normalised to 1 at large distances. In the rainbow region the survival probability is $\sim 3 \cdot 10^{-3}$ at a $L$-value of 40 .

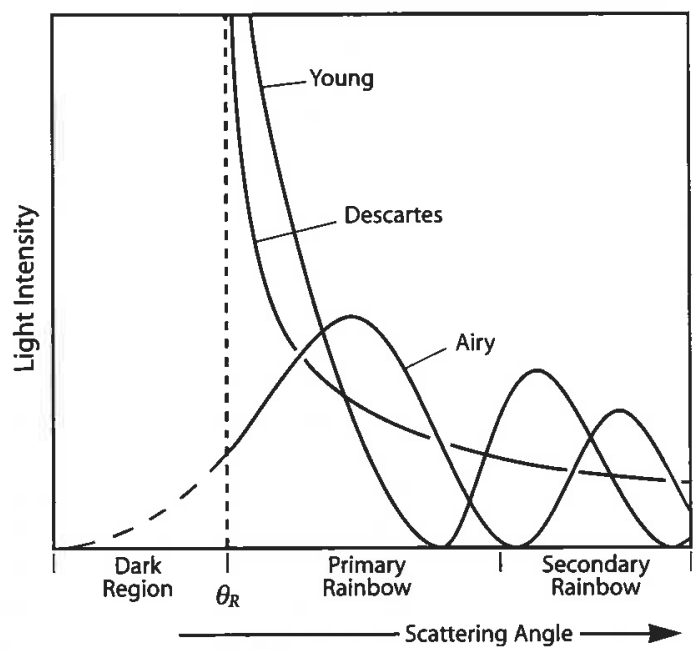

Fig. B1.2 The functional dependence of the light intensity at the maximum deflection angle. This angle defines classically the separation between light and shadow. The correct description is given by Airy functions, which place the intensity maximum at angles inside the "lighted" region and exhibits oscillations, to which we will refer as primary or secondary (and higher) Airy maxima and minima (marked as A1, A2 etc. in figure 3). The scattered intensity now extends into the classically forbidden region (dashed lines). The other curves denoted by Young and Descartes show unphysical singularities at $\theta_{R}$, which were later resolved by Airy.

rainbow in heavy ion scattering in the elastic scattering for ${ }^{16} \mathrm{O}+{ }^{16} \mathrm{O}$ at $350 \mathrm{MeV}$ the rainbow maximum at an angle of $\theta_{C M} \approx 50^{\circ}$ turned out to be the primary Airy maximum (see also figs. 2 and 3 ). Subsequent analysis by many authors [2] revealed that rather deep potentials are needed to describe the experimental result. In the following years systematic experimental studies of the ${ }^{16} \mathrm{O}+{ }^{16} \mathrm{O}$ system over a wide range of energies have been performed. Now high precision data [3] exist for angular distributions over a wide angular range from low energies at $E_{L}=75-100 \mathrm{MeV}$, measured in small energy intervals at IreS Strasbourg, as well as at high energies from $10 \mathrm{MeV} / \mathrm{u}$ up to $70 \mathrm{MeV} / \mathrm{u}$ measured at the HMI and at GANIL (see Figs. 1 and 2).

The cover figure (fig. 1) shows an overview of the data plotted as function of the momentum transfer $q R$, as already explained. This presentation helps to emphasise the wave properties of the scattering. In this figure two remarkable features become apparent:

a) the diffractive regime dominates at small momentum transfer. The pattern changes very little over the range of energies with 10-30 MeV/nucleon.

b) The nuclear rainbow region appears at larger $q R$ values. The dependence of the position of the primary rainbow angle on incident energy is well documented by these data. The rainbow angle decreases with increasing incident energy, because the real potential is only slightly dependent on energy; the refractive index $n=\lambda_{0} / \lambda$ approaches unity for large energies. At $E_{L}=704 \mathrm{MeV}$ the rainbow maximum is already less pronounced; at an energy of $1.12 \mathrm{GeV}$ the refractive and diffractive regions start to merge.

Inspecting Fig. 2 it is interesting to follow the position of the nuclear rainbow angle, $\theta_{R}$, to smaller energies. At the energy $E_{L}=704 \mathrm{MeV}, 480 \mathrm{MeV}$ and $350 \mathrm{MeV}$ the primary Airy maximum is well observed as a bump on the decreasing differential cross section which appears after the diffractive pattern. At $250 \mathrm{MeV}, \theta_{R}$ has moved to angles larger $\theta_{C M}=90^{\circ}$ and in the region of the measured data points the secondary Airy maximum appears. A de- tailed study of the low energy data at $E_{L} \leq 125 \mathrm{MeV}$ has shown that only the deep potentials give the correct interpretation of the data, in a smooth continuation from the unique determinations of the potentials at $E_{L}=350 \mathrm{MeV}$ and $480 \mathrm{MeV}$. The pronounced structures in the angular distributions recently measured at IreS (Strasbourg) at even lower energies down to $E_{L}=75-80 \mathrm{MeV}$ must be interpreted as manifestations of refractive scattering with the $3^{\text {rd }}$ and $4^{\text {th }}$ Airy minima moving through the region of $\theta_{C M}=90^{\circ}$. As shown in fig. 3 for the case of $E_{L}=145 \mathrm{MeV}$, the pronounced structures observed at this energy are clearly identified as higher order Airy structures. In this figure the interference structure, which appears around $\theta_{C M}=90^{\circ}$ for identical bosons has been removed for clarity by making a nonsymmetrized calculation.

These data, with the systematic analysis up to the highest energies give a clear answer to an old problem of heavy ion science, namely the interpretation of the pronounced structures observed in the excitation functions of heavy ion scattering at low energies, specifically for ${ }^{12} \mathrm{C}+{ }^{12} \mathrm{C}$ and ${ }^{16} \mathrm{O}+{ }^{16} \mathrm{O}$. The behaviour of the elastic scattering cross section at $\theta_{C M}=90^{\circ}$ as function of incident energy has been a matter of debate in the 1970 s and 1980 s because "resonances" were considered to be at the origin of the pronounced maxima and minima. Minima at $\theta_{C M}=90^{\circ}$ for bosons with spin $=0$ (the ${ }^{16} \mathrm{O}$-nuclei) were in fact difficult to reproduce in the optical model calculations, with shallow potentials at that time. We now understand the origin of these minima in the cross sections as the sequence of very sharp higher order Airy minima, which move through $\theta_{C M}=90^{\circ}$ as the energy is decreased. The refractive scattering is thus observed down to quite low energies of $5 \mathrm{MeV} / \mathrm{u}$.

\section{The soft equation of state}

Finally we come back to the properties of the equation of state around the saturation point $\rho_{o}=0.17 \mathrm{fm}^{-3}$. Refractive nuclear scattering of strongly bound light ions, in particular $\alpha$-particles, ${ }^{12} C$-ions and ${ }^{16} \mathrm{O}$-ions cover the region of density overlaps up to $\rho \approx 2 \rho_{0}$; actually the properties of the nucleon-nucleon interaction in medium is probed, which in turn is used to calculate properties of nuclear matter. This procedure is also well known from studies of high energy central collisions, in which reactions with many particles in the final channel are modelled using different $\mathrm{N}-\mathrm{N}$ interactions and compared to various experimental observables (flow, 


\section{Box 2 The equation of state of cold nuclear matter}

The equation of state of nuclear matter describes the variation of the total energy in its dependence on the density $\rho$, or more specifically for a finite system, the binding energy per nucleon, as function of density (and temperature). Fig. B2.1 shows the variation of this quantity over a region of densities $\rho / \rho_{o}=0.0$ to 4.0 relative to the saturation value $\rho_{0}$. The saturation point of nuclear matter is given by $E / A=17.2 \mathrm{MeV}$ at $\rho_{o}=0.17 \mathrm{fm}^{-3}$. The variation of $E / A(\rho)$ around $\rho_{o}$ can actually be well described by a quadratic function. The examples shown in fig. B2.1 have been calculated with an effective nucleon-nucleon interaction which is density dependent, which allows: $i)$ to reproduce the correct saturation point at $\rho_{0}$, and, ii) gives various curvatures around the saturation point, the second derivative defines the incompressibility parameter $K$. The system is said to be soft for values of $K$ in the range of $150-250 \mathrm{MeV}$.

Other studies on the determination of the incompressibility are based on the study of the nuclear monopole excitation (density vibrations) observed in nuclear inelastic scattering. A more recent analysis of well chosen examples ( $\mathrm{Sn}$ and $\mathrm{Pb}$ nuclei) gives a value of $K \approx 220 \mathrm{MeV}$, corresponding to a "soft" equation of state. We note, however, that the density variation of nuclear matter in the monopole vibration is only a few percent of $\rho_{0}$.

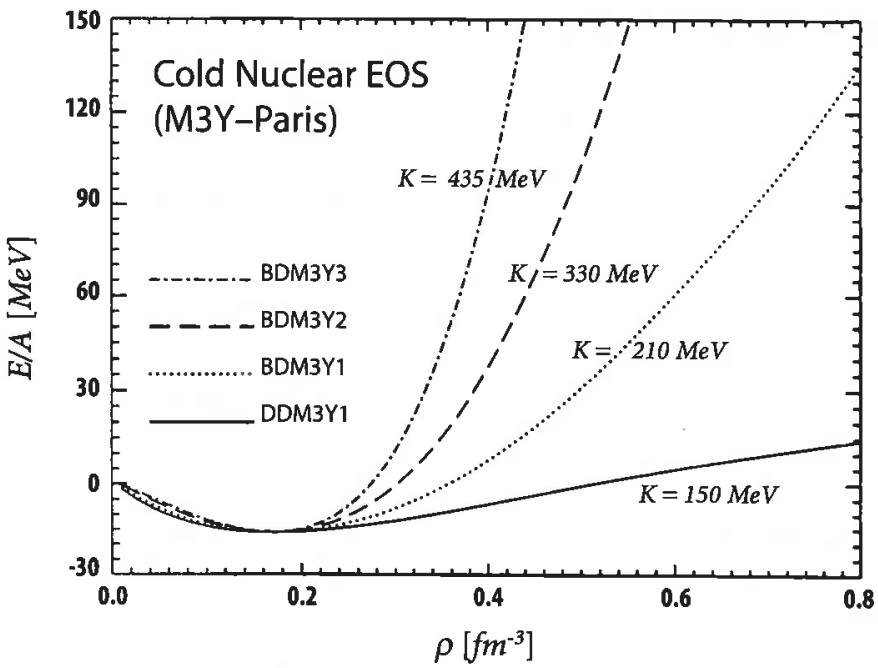

Fig. B2.1 Binding energy per nucleon, $E / A(\rho)$, as function of density $\rho$ for nuclear matter as obtained from Hartree-Fock calculations using the effective nucleonnucleon interaction of the "Paris-type" with a density dependence chosen so as to reproduce the correct saturation energy of nuclear matter at $\rho=\rho_{o}$, for various values of the incompressibility parameter $K$. A small density dependence is needed in order to obtain saturation; a rather strong density dependence will lead to a stiff EOS with $K \geq 400 \mathrm{MeV}$ and to less deep potentials in the folding model. The data on ${ }^{16} \mathrm{O}+{ }^{16} \mathrm{O}$ scattering test regions up to $2 \rho_{0}$ and give a soft equation of state with $K \approx 250 \mathrm{MeV}$.

energy spectra of fragments, fragment mass distribution). However, these studies are related to hydrodynamical modes with compression and to hot nuclear matter.

For the studies of refractive rainbow scattering in the well defined elastic channel, the very high quality data give access to so called model independent methods for the determination of the underlying real and imaginary parts of the optical model. The potentials are obtained with inversion procedure.

We deduce from our analysis a value of $K=250 \mathrm{MeV} \pm 25 \mathrm{MeV}$ [3], the precision, with which the incompressibility can be determined is about $10 \%$. We find that cold nuclear matter is "soft", which is in agreement with a variety of other recent studies on monopole excitations of nuclei.

\section{Acknowledgements:}

This study has been done in close collaboration with the University of Tuebingen and with GANIL (Caen, France). We are indebted to various institutions for financial support: The A.v. Humboldt Foundation, the BMBF (Verbundforschung Kontrakt-Nr. 060B115), the DFG (Graduiertenkolleg of UNI Tuebingen), the European TMR program (Access to Large Facilities at GANIL).

\section{Of Colours}

Among the various phenomena of colours, there is none more remarkable than that of the rainbow, which is an appearance observable in those places only where it rains in the sunshine, and where the spectator is placed in a due position between the sun and the rain, with his back to the former; for which reason is generally allowed, that the bow is made by the refraction of the sun's light in drops of falling rain; the manner wherin is formed, has some measure been explained by ANTONIUS DE DOMINIS, archbishop of Spalato, and after him by DES CARTES; but neither of them understood.

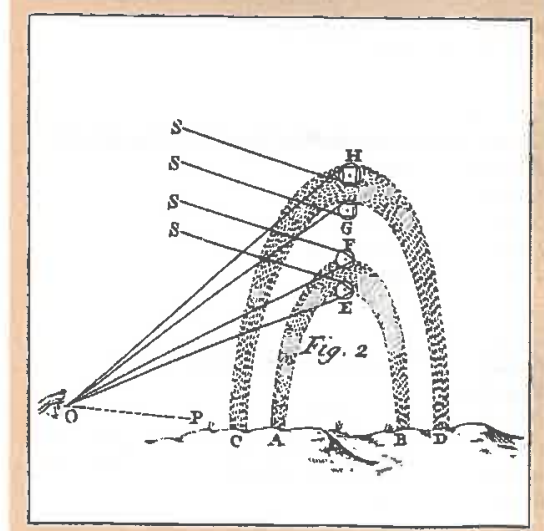

Taken from Lectures on Natural History, Richard Helsham 1739 an error bound, which is related to the precision of the data, and the resulting potentials can directly be compared to those obtained in folding models showing perfect agreement with the theoretical prediction for a particular choice of the density dependence of the nucleon-nucleon interaction. This is a unique feature of the elastic channel in which a well defined physical quantity, measured with high precision, can be related to theory by a mathematical 